\title{
Digital Household Energy Meter with GSM Communication for Home Energy Management
}

\author{
Samudra De Silva ${ }^{\# 1}$, Yasantha C. Samarawickrama ${ }^{\# 2}$, D. C. Jayasekara ${ }^{\# 3}$ \\ ${ }^{\#}$ Faculty of Engineering and Technology, CINEC, Millennium Drive, IT Park, Malabe, Sri Lanka. \\ ${ }^{1}$ samudradesilva@gmail.com \\ ${ }^{2}$ yasanthalcinec.edu \\ ${ }^{3}$ deepesh.jayasekara@cinec.edu
}

\begin{abstract}
This design is developed with the main aim of assisting energy consumers and utility service providers with an enhanced feature for better home energy management. Automatic generation of the monthly electricity bill avoids the prevailing drawbacks of the current energy measuring system such as time wastage, accuracy issues, human errors and possibility for fraud activities. This system can be effectively used for real time measuring and analyzing of the household energy usage. The device utilizes GSM technology to alert the consumers with energy readings in Kilo Watt hours (kWh). Any individual or an organization with an approved electricity connection by the standard utility service provider can use this device. Crucial parameters of energy appliances such as Power Factor, Rated Power and Root Mean Square (RMS) Current were analyzed with the design obtaining results appropriately. Demand charge can be effectively controlled avoiding the overall household demand exceeding a preset demand value. As evident from the specifications and results this study fulfills a prudent requirement in the home energy management sector.
\end{abstract}

Keywords - Digital Energy Measurements; Low cost, Home Energy Management; Power Factor; Peak Demand Controlling

\section{INTRODUCTION}

The designed device as proposed in this paper calculates the monthly electricity bill automatically and communicates the payment amount to the consumers' mobile device at the end of each payment period. Generally, an authorized person from $\mathrm{CEB}$ visits every house for the meter reading activity. But, this method creates disadvantages such as wastage of time, accuracy issues of meter reading and the ability for customers and the meter readers to engage in any fraudulent activities. Moreover, the system proposed by this paper requires less labor since the energy metering device carries out the job role of the meter reader. This introduction of "Digital Energy Metering System" empowered through GSM communication shall do the job accurately and is convenient for both CEB and consumers due to automation of the system.

The current traditional energy metering devices installed at households consists of a lot of moving mechanical parts causing enhanced complexity to troubleshoot and debug the errors. However, the proposed energy meter consists less mechanical parts causing troubleshooting and debugging to be easier than in the traditional energy meter. Further, the digital energy meter was designed based on Atmega328P microcontroller with various novel features that can be equipped in comparison to the traditional energy metering devices currently in operation.

\section{II.LITERATURE REVIEW}

\section{A. Digital energy metering system}

In the present context, the bill is processed by the meter reader who visits the premises and goes through a manual process which clearly ascribes the probability of human errors. This process is reported as unreliable [1]. In case if the electricity consumer forgets to settle the bill, the service provider will disconnect the electricity without any hesitation [2]. Thus, to overcome aforementioned disadvantages an energy meter which can monitor and alert the energy usage digitally in real-time to consumers was proposed and designed.

\section{B. Background Study}

Recent approach by Kurkute, et al. [1] proposes an energy metering device with an Atmega16 microcontroller facilitated with low power consumption in 2016. Similarly, the same concept was bought forward by Gowsami and Hasarika [3] prior to Kurkute, Girase and Patil [1] in 2015. However, the less concern about the accuracy and the inability of communicating the measurements to a remote location have been major drawbacks of these systems.

Jose, et al. [4] proposed a smart energy meter with a PIC16F877A microcontroller and a MAX232 communication system which interfaces the microcontroller with the GSM modem. Moreover, a digital energy meter with an ARM LPC2119 microcontroller which is not commonly used in modern applications with IR transmitters and receivers has been proposed to use by Silviya, et al [5] in their journal paper which was published in 2014.

Another research was carried out by Siveram [6] in 2013. But the main feature in his proposed system is the usage of AT7751 energy monitoring IC and an Atmega8 microcontroller. The major drawback of this proposed system was the usage of an Atmega8 microcontroller containing limited input and output pins in the work space.

In addition, another rare type of an energy meter was built by Satheyamoorthy (2013) [7] using a PLC system. There are various disadvantages in designing a meter reading device using PLC due to its high initial cost and the complexity of programming environment. Moreover, unlike in Arduino PLC, this should be programmed using Ladder Logic, Function Block Diagram or List of instructions which is not userfriendly. The troubleshooting of this system requires high end expertise knowledge and special training as well. Further, libraries assisting for programming Arduino are not available in the PLC system. Hence, these facts depict that, designing an 
energy meter reading system using PLC is not an effective approach.

Similarly, the microcontroller AT89S52 has been used in a project of prepaid energy metering system. A prepaid energy meter is an energy metering system that the consumer pays the supplier initially and when the paid credit limit is reached a SMS is sent to the consumer's mobile device through the GSM module alerting the usage of energy. This approach assists for the consumption of the energy with a control [8][9] reducing the overall energy cost in the household environment. However, accuracy of the energy measurements and the userfriendliness of the system is found to be in a very low state in this design. Further, Keerthi and Raju (2015) [10] designed a Digital energy metering system using MSP430F5529 series microcontroller due to its ultra-low power features. This series of microcontrollers offer a wide range of control features at a cost of more complexity in design.

Considering all the related background work, an outstanding research gap is observed to design a low cost Digital Energy Meter with highly accurate measurements in addition of features such as GSM communication and peak demand controlling. The authors have decided to utilize Arduino technology with user-friendliness in programming and troubleshooting with the accessibility to a variety of libraries. Real time Energy monitoring feature and user alerts is fulfilling a very prudent requirement in the modern world Energy industry.

\section{METHODOLOGY}

This chapter focuses about the hardware design of the proposed and designed digital Energy meter and the required calculations performed to achieve the required features of the design.

\section{A. Block diagram of the designed system}

The figure depicted in the fig. 1 illustrates the functional block diagram of the system which was developed.

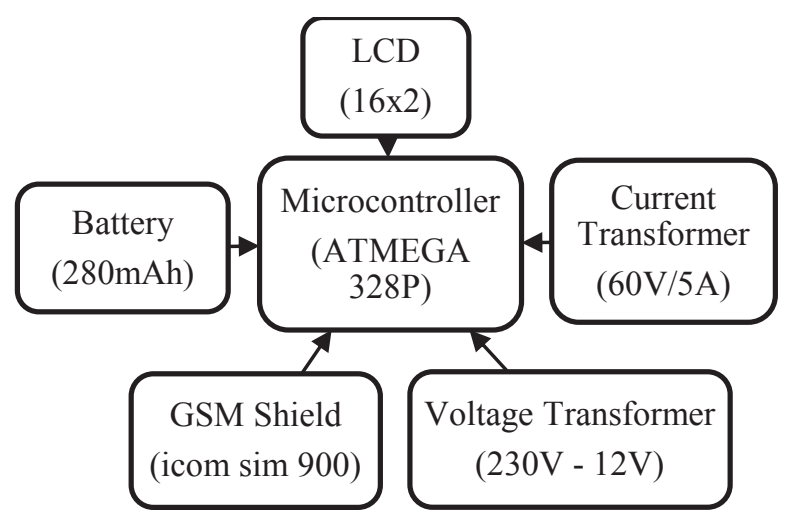

Fig. 1 Block diagram of the developed system.

$230 \mathrm{~V}-12 \mathrm{~V}$ step down voltage transformer was used in the design for two main purposes. The first main purpose was the stepping down of the voltage from the main grid to be provided as an input to the processing unit. Other purpose was to generate a pulse thus the Atmega328P microcontroller enabling the detection of the voltage in the grid at that time. Current transformer of $60 \mathrm{~A} / 5 \mathrm{~A}$ was used to detect the current drawn by the electrical appliance plugged in to the digital energy meter. The objective of utilizing a LCD display in the following device is to display the required parameters such as Supply voltage, Supply current, Power factor and Energy usage in real time.

An Atmega328P microcontroller was used in the device as the main processing unit. This microcontroller was interfaced with all the other input and output components used in the design. Current transformer used to sense the current generates a pulse and transmit it to the Atmega328P via analog pins. Similarly, voltage transformer also generates a pulse and transmit it to Atmega328P microcontroller. Thus, the values received by the Atmega328P microcontroller is conveyed to consumer through the LCD display equipped in the design. Further, the device is facilitated with a GSM shield enabling SMS communication to consumers acknowledging about the monthly energy consumption cost of the household premises.

A battery of $280 \mathrm{mAh}$ is used in the device to fulfil the power requirements. Ensuring the integrity of the saved data of the calculations, a rechargeable battery was used instead of a normal battery.

\section{B. Design Calculations}

Several calculations were carried out to compute the parameters of the design achieving required specifications.

\section{1) Voltage Transformer Calculations:}

\section{- Calculation of number of turns}

If the number of turns in the primary and the number of turns in the secondary is denoted as $N_{p}$ and $N_{s}$ respectively while Voltage in the primary and the voltage in the secondary is $V_{p}$ and $V_{s}$ respectively,

$$
\begin{aligned}
& N_{S}=\frac{N_{P} \times V_{S}}{V_{P}} \\
& N_{S}=\frac{360 \times 12 \mathrm{~V}}{230 \mathrm{~V}} \\
& N_{S}=18.78 \text { turns }
\end{aligned}
$$

Hence, the number of secondary turns available in the transformer is practically obtained as 19 approximating 18.78 value.

\section{- $\quad$ Calculation of the input current}

If the current in the primary and secondary is given by $I_{p}$ and $I_{s}$, we can use the following relationship to calculate the amount of current drawn by the primary of the voltage transformer.

$$
\begin{gathered}
\frac{N_{P}}{N_{S}}=\frac{V_{P}}{V_{S}}=\frac{I_{S}}{I_{P}} \\
I_{P}=\frac{I_{S} \times V_{S}}{V_{P}} \\
I_{P}=\frac{1 \mathrm{~A} \times 12 \mathrm{~V}}{230 \mathrm{~V}} \\
I_{P}=0.052 \mathrm{~A}=52.1 \mathrm{~mA}
\end{gathered}
$$


Therefore, the voltage transformer draws $52.1 \mathrm{~mA}$ current to step down $240 \mathrm{~V}$ to $12 \mathrm{~V}$ with a maximum current output of 1A through the secondary winding according to the above calculation.

\section{- Calculation of the Power dissipation}

The following equation can be employed to calculate the power dissipation of the primary of the transformer.

$$
\text { Power }_{\text {primary }}=V_{P} \times I_{P}=230 \mathrm{~V} \times\left(52 \times 10^{-3}\right) \mathrm{A}=11.96 \mathrm{~W}
$$

Power dissipation is calculated as 11.96 Watts. Thus, it can be rounded off and stated as 12 Watts. However, to clarify the above answer the power dissipation in the secondary shall be calculated. Using the assumption that the voltage transformer is an ideal transformer where there is no power loss, the following equation can be derived.

$$
\begin{gathered}
\text { Power }_{\text {primary }}=\text { Power }_{\text {secondary }} \\
\text { Power }_{\text {secondary }}=V_{S} \times I_{S}=12 \mathrm{~V} \times 1 \mathrm{~A}=12 \mathrm{~W}
\end{gathered}
$$

Hence, the power dissipation of the voltage transformer is $12 \mathrm{~W}$ from the above calculations.

\section{- Calculation of the Transformer Efficiency}

The conversion efficiency of the voltage transformer can be found by using the following relationship.

$$
\begin{gathered}
\text { Efficiency }=\frac{\text { Output Power }}{\text { Input Power }} \times 100 \% \\
\text { Efficiency }=\frac{11.96 W}{12 W} \times 100 \% \\
\text { Efficiency }=99 \%
\end{gathered}
$$

Hence, the efficiency of the transformer is calculated as $99 \%$. Usage of a transformer with high efficiency can lead to gain precise readings. In a digital energy metering design, low power consumption of the design and usage of a highly efficient transformer in order to map the pulse to be provided as input for main controlling unit is essential.

\section{2) Calculations related to current transformer:}

\section{- $\quad$ To calculate the number of Secondary Windings}

If the Current in the primary and the Current in the secondary is $I_{p}$ and $I_{s}$ respectively, the following relationship can be derived with the number of turns in the primary and secondary.

$$
\begin{array}{lr}
\frac{N_{P}}{N_{S}}=\frac{I_{S}}{I_{P}} & N_{S}=\frac{1 \times 60}{5} \\
N_{S}=\frac{N_{P} \times I_{P}}{I_{S}} & N_{S}=12 \text { turns }
\end{array}
$$

The ratio of the current transformer used in the design to interface with the digital energy metering device is 60:5. Using this specification, the number of turns in the secondary is calculated as 12 turns according to the above relationship.
Similarly, to calculate the current drawn by each appliance tested during the testing procedure the following equation was used.

$$
\text { Current Drawn }=\frac{\text { Rated power of the appliance }}{\text { Operating voltage }}
$$

Power factor was calculated using the power triangle. Therefore, the equation used to calculate the power factor is as following.

$$
\begin{gathered}
\text { power Factor }=\frac{\text { Real Power }(k W)}{\text { Apperant Power }(k V A)} \\
\text { IV. RESULTS }
\end{gathered}
$$

The operation of the device was tested appropriately using several tests. For testing purposes three filament bulbs of $40 \mathrm{~W}$, $60 \mathrm{~W}$ and $100 \mathrm{~W}$ were used as electrical appliances. These appliances were investigated for a range of tests of real power, testing for $\mathrm{I}_{\mathrm{RMS}}$ and power factor. The results certified the device was operating according to the specifications.

\section{A. Testing the Real power}

The sources of energy were tested employing the digital energy meter in identifying if the rated power is mentioned accurately in the appliances. During the test, the appliances were fixed in to the digital energy meter in obtaining the reading of the rated power.

Fig. 2 illustrates the variation of the real power in Watts against time. It can be concluded that there are minor deviations of the real power and the appliance does not always deliver the rated wattage value. This is mainly due to the specified tolerance level of the manufacturers. This design can be effectively used to test the electrical appliances for their actual power consumptions and the accuracy of the specified tolerance.

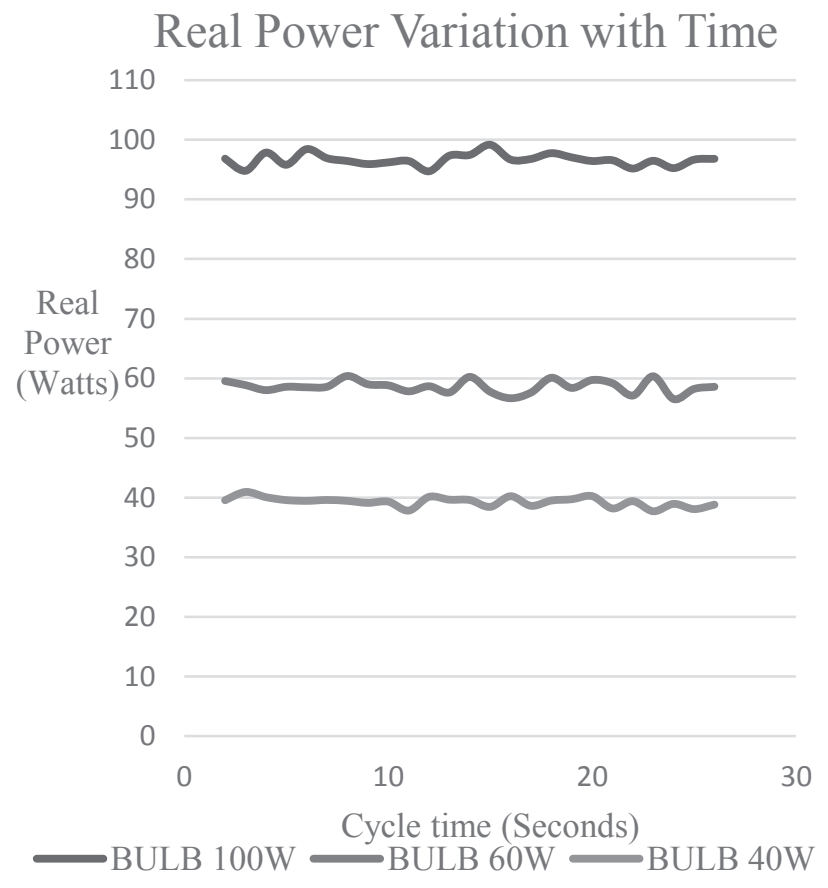

Fig. 2 Real Power Measurements of appliances against Time 


\section{B. Testing the IRMS value}

This test is conducted to determine the $\mathrm{I}_{\mathrm{RMS}}$ value of the appliance. In conducting this test same bulbs of $100 \mathrm{~W}, 60 \mathrm{~W}$ and $40 \mathrm{~W}$ were used. Thus, it was identified that $0.44 \mathrm{~A}, 0.27 \mathrm{~A}$ and $0.18 \mathrm{~A}$ will be the $\mathrm{I}_{\mathrm{RMS}}$ values for $100 \mathrm{~W}, 60 \mathrm{~W}$ and $40 \mathrm{~W}$ bulbs respectively. The results were justified from the current drawn equation mentioned in the calculations section.

Therefore, graphical representation was used to analyze the results obtained while conducting the above test is illustrated in Fig. 3. However, 0.01 Amperes deviation was observed analyzing the graphs plotted after computing the current drawn by the appliance.

These results prove that this design can be effectively used to analyze the current drawn from each appliance and ultimately troubleshoot and find out if any malfunctions present in the device.

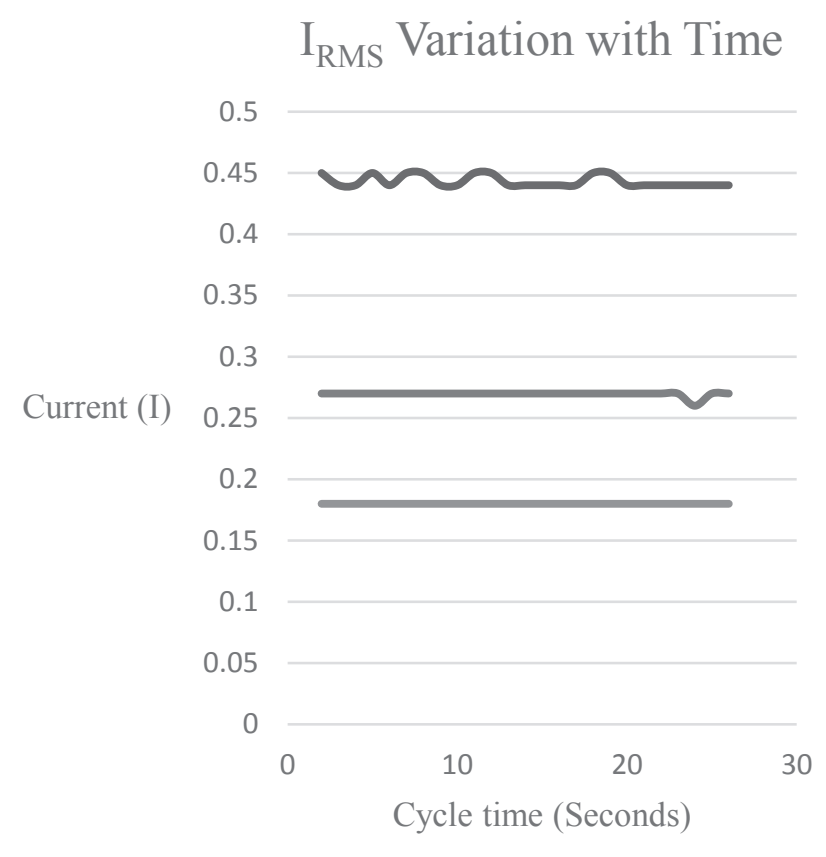

\section{$\longrightarrow$ BULB 100W $\longrightarrow$ BULB 60W $\longrightarrow$ BULB 40W}

Fig. $3 \mathrm{I}_{\text {RMS }}$ Readings of Appliances against Time

\section{Testing the Power Factor}

The power factor is a crucial parameter to be measured to get an idea about the efficiency of the device under operation. The Power Factor was calculated by multiplying the real power by the apparent power. Power factor in ideal operation was considered to be 1 making the appliance work with an efficiency of $100 \%$. Hence, to analyze the efficiency of the appliances the power factor was graphically represented for the same bulbs used in the earlier tests and compared with the ideal power factor of 1 .

Fig. 4 below illustrates the variation of the power factor against time. The $100 \mathrm{~W}$ bulb was reported to operate with a maximum efficiency of $95.12 \%$. Similarly, $60 \mathrm{~W}$ bulb demonstrated an efficiency of $94.92 \%$ while $40 \mathrm{~W}$ bulb reported an efficiency of $94.76 \%$. This results in generating the power factor values highlight the possible usage of the design for home energy management applications which is an upcoming trend and a prudent requirement in the modern world.

\section{Power Factor Variation with Time}

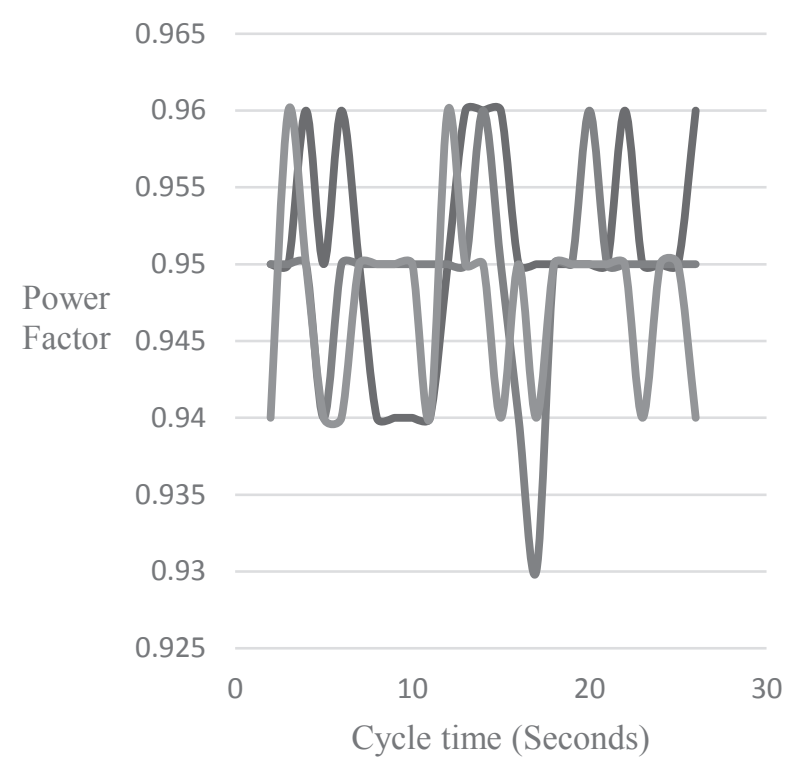

$\longrightarrow$ BULB 100W $\longrightarrow$ BULB 60W - BULB 40W

Fig. 4 Power Factor variation against Time

V. Costing OF Design

TABLE I

COSTING OF THE DESIGN

\begin{tabular}{|c|c|}
\hline Description of the Item & Cost (LKR) \\
\hline Atmega328P microcontroller & 250.00 \\
\hline SIM900 GPRS/GSM Shield & 6000.00 \\
\hline LCD Display - 16x2 260.00 \\
\hline Voltage transformer (230v - 12v) & 850.00 \\
\hline Current transformer (60A:5A) & 1300.00 \\
\hline Wires & 550.00 \\
\hline Multi - Socket plug base & 750.00 \\
\hline Rechargeable battery of 9v & 1115.00 \\
\hline Miscellaneous & 2250.00 \\
\hline Total & 13325.00 \\
\hline
\end{tabular}

The low-cost feature of the design was a great achievement which fulfils a current market requirement. The total budget for the entire design was LKR 13,325 ensuring quality and accurate components meeting the required specifications as well as standards. Hence, it can be concluded that the design has achieved the low-cost feature costing less than 90 USD. This cost can be further reduced in the scale of mass production. 


\section{DISCUSSION AND CONCLUSION}

The digital energy metering device designed is a huge success achieving outstanding low-cost features compared to other similar caliber of products in the current market. The device generates real time data for better understanding of the energy consumption at the household premises. This assists home energy management for the customers and utility service providers. The ability to analyze the rated power, $\mathrm{I}_{\mathrm{RMS}}$ and the Power factor of the electrical appliances enhance the importance of this device further. It is also possible to use this device for Peak demand controlling feature trimming the high demand cost. This extends the usability of the device from the household sector to the industrial sector as well.

Moreover, the energy monitoring device replaces traditional electricity bill calculation method overcoming multiple drawbacks. This is achieved by sending SMS messages to the consumer alerting on the usage of the bill at the end of each payment period. The device displays the realtime energy usage by the LCD display mounted on top of the device.

The device has been manufactured with easy clip on method. Thus, the device can be connected to the appliances with a user-friendly manner. Hence, it saves time of the customers with easiness to setup feature. The ability to use the device practically in investigating real power consumption of the electrical appliances provides the feature to compare the practical energy consumption with the rated value providing tolerance details of the power consumption of appliances.

\section{FUTURE DEVELOPMENTS}

This design can be considered as an outstanding achievement with aims and objectives achieved as planned. This fulfils a timely requirement in the home energy management field. However, following modifications can be considered for further development of the device avoiding some drawbacks currently present in making the device much more user friendly to the energy consumers and utility service providers.

There is a possibility to develop an I2C communication system to interface the LCD display with the Atmega328P microcontroller. Hence the wiring can be further reduced making the device easy to use and troubleshoot. Moreover, another suggested development for the aforementioned device is programming the energy meter using high end languages and incorporating Raspberry Pi or Linux based system with much more additional features. This device can be incorporated with SMART HOME concept providing the feature to control the appliances to achieve optimum energy consumption required leading to better home energy management.

In the current design only one mobile device can be interfaced with the GSM Shield. Hence, it is suggested to allow multiple mobile devices to interface with the GSM shield of the energy monitoring device. This enables to communicate with multiple subscribers including energy consumers and energy utility service providers providing better information exchange as alerts. Developing a web based Graphical User Interface (GUI) for the energy monitoring device can be suggested as well as incorporating high-end security protocols. This will allow the consumer to monitor real-time energy usage over the internet using an e - portal.
Outcome of this research is highly satisfactory as the design fulfills a prudent requirement especially in third world countries including Sri Lanka. Further research will be carried out incorporating above suggested modifications to facilitate consumers with better features and enhanced usability.

\section{REFERENCES}

[1] Kurkute, S.R., Girase, G. and Patil, P. (2016). 'Automated Energy Meter Reading System Using GSM Technology', International Journal of Innovative Research in Electrical, Electronics, Instrumentation and Control Engineering, pp. 149-152. DOI 10.17148/IJIREEICE.2016.

[2] Rao, V.K and Madhu, S.G.N. (2013). 'GSM Based Energy Meter Reading and Billing', IJSR, 5(8), pp. 683-687.

[3] Gowsami, A. and Hazarika, A. (2015). 'GSM Based Automatic Energy Meter Reading', International Journal of Innovative Research in Science, Engineering and Technology, pp. 12860-12864. DOI:10.15680/IJIRSET.2015.

[4] Jose, J.K., Mohan, L., Nijeesh, U.K. and Benny, T.C. (2015). 'Smart energy Meter'. International Journal of Engineering Trends and Technology, 22(4), pp. 179-182.

[5] Silviya, M.E., Vinodhini, M.K. and ThilagamJ, S.T. (2014) 'GSM Based Automatic Energy Meter System with Instant Billing’, International Journal of Advance Research in Electrical, Electronics and Instrumentation Engineering, 39(3), pp. 44-49.

[6] Siveram, S. (2013). 'GSM Based Smart Energy Meter with Theft Detection and Fault Identification'. International Journal of Science and Research, 4(9), pp. 603-605.

[7] Satheyamoorthy, K.P. (2013). 'Smart Energy Meter Load Control'. International Journal of Advance Research in Electrical, Electronics and Instrumentation Engineering, 2(8), pp. 3845-3851.

[8] Jubi, K. and Mareena, J. (2013). 'Prepaid Energy Meter with GSM Technology', American International Journal of Research in Science, Technology, Engineering and Mathematics, pp. 195-198.

[9] Upadhyay, J., Devadiga, N., D'mello, A. and Fernandez, G. (2015). 'Prepaid Energy Meter with GSM Technology', International Journal of Innovative Research in Computer and Communication Engineering, pp. 2048-2054. DOI 10.15680/IJIRCCE.2015.

[10] Keerthi, M. and Raju, N. (2015). 'Automated Utility Meter Reading Using Wireless System Bluetooth with MSP430 Microcontroller'. International Journal of Emerging Engineering Research and Technology, 3(11), pp. 39-45. 